\title{
Developing and testing a miniature fiber-coupled scintillator for in-core neutron counting in CROCUS
}

\author{
Fanny Vitullo*, Vincent Lamirand*, Jean-Baptiste Mosset $^{\dagger}$, Pavel Frajtag*, \\ Oskari Pakari*, Gregory Perret ${ }^{\dagger}$ and Andreas Pautz ${ }^{*} \dagger$ \\ *École polytechnique fédérale de Lausanne, Lausanne, Switzerland \\ ${ }^{\dagger}$ Paul Scherrer Institut (PSI), Villigen, Switzerland \\ Email: fanny.vitullo@epfl.ch
}

\begin{abstract}
An advanced neutron detection system for highly localized measurements in nuclear reactor cores was developed and tested in the Laboratory for Reactor Physics and System Behaviour (LRS) at the École polytechnique fédérale de Lausanne (EPFL), Switzerland, in close collaboration with the Detector group of the Laboratory for Particle Physics (LTP) at the Paul Scherrer Institute (PSI), Switzerland. The miniature-size detector is based on the coupling of a $\mathrm{ZnS:}{ }^{6} \mathrm{LiF}$ scintillator/converter screen of $1 \mathrm{~mm}^{2}$ and $0.2 \mathrm{~mm}$ thickness with a $10-\mathrm{m}$ optical fiber, the latter being connected to a silicon photomultiplier (SiPM). In this development version, the output signal is processed via analog read-out electronics. The present work documents the characterization of a detection system prototype in the mixedradiation fields of $t$ he $C A R R O U S E L$ acility a nd $i$ ts $t$ esting in the CROCUS zero-power reactor operated at LRS. The fibercoupled scintillator shows a linear response with the reactor power increase up to $6.5 \mathrm{~W}$ (i.e. around $10^{8} \mathrm{~cm}^{-2} \cdot \mathrm{s}^{-1}$ total neutron flux), w ith a $s$ ubsequent $l$ oss of $l$ inearity d ue $t$ o e lectronic dead time of the analog system. Nevertheless, the detector shows excellent neutron counting capabilities whether compared to other localized detection systems available at LRS, e.g. miniature fission chambers and an SCVD diamond detector.

Keywords-scintillator, in-core neutron detection, optical fibers, CROCUS reactor.
\end{abstract}

\section{INTRODUCTION}

Neutron measurements with high spatial resolution are paramount for the investigation of complex in-core phenomena and for the validation of modern high-fidelity neutron transport codes. Highly-resolved experiments have been performed in the past in the zero-power research reactor PROTEUS, operated at Paul Scherrer Institut (PSI) from 1968 to 2011, by means of neutron activation techniques [1], [2], [3] or standard reactor instrumentation like miniature fission chambers [4]. The idea to perform extensive investigations of threedimensional static and dynamic in-core phenomena in research reactors [5], [6] triggered the development of advanced neutron detection techniques for highly localized measurements. These applications require instruments with high sensitivity and flexibility, a s w ell a s t he p ossibility $\mathrm{t}$ o b e p arallelized and to perform online measurements. Scintillation materials have recently come to the fore in the field of neutron detection [7] due to the large availability of screens of reduced size with good efficiency a nd $l$ ow $p$ rice, $t$ ogether $w$ ith the possibility to couple them with cutting-edge light collection technologies and fast electronics. A first detection system prototype for incore applications using a scintillator/converter $\mathrm{ZnS}:{ }^{6} \mathrm{LiF}$ screen was developed at Nagoya University in 1994 by Mori et al. [8] and tested in the Studsvik-Nuclear $1 \mathrm{~W}$ reactor [9]. However, the scintillator was connected with an optical fiber to a photomultiplier tube (PMT) that limited the possibility of parallelization of the designed detector. Over the past decade photodetector technologies have seen a shift from the use of classical PMTs to solid-state devices such as silicon photomultipliers (SiPMs), which are robust, compact and electronically stable instruments [10]. The coupling of a $\mathrm{ZnS}:{ }^{6} \mathrm{LiF}$ scintillator with a SiPM has been already tested by the Detector group of the Laboratory for Particle Physics (LTP) at PSI, Switzerland, in the framework of the upgrade of a neutron diffractometer [11], [12]. Although this detection system was not developed for in-core reactor applications, an adapted version of the latter would represent an efficient solution to perform highlyresolved experiments in research reactors and to engineer advanced non-invasive reactor diagnostic techniques [13].

In the current work a cutting-edge neutron detection system has been designed for the investigation of highly localized in-core phenomena in the CROCUS zero-power research reactor [14], operated by the Laboratory for Reactor Physics and System Behaviour (LRS) at the École polytechnique fédérale de Lausanne (EPFL), Switzerland. The advanced system, built in collaboration with the LTP group at PSI, features a miniature $\mathrm{ZnS}:^{6} \mathrm{LiF}$ scintillator coupled to a SiPM via a $10-\mathrm{m}$ optical fiber. An analog read-out electronics is employed for signal processing. The present work documents the characterization and testing of the new neutron detection system. The first part of the paper is dedicated to a detailed description of the detection system and its components. The second part of the paper presents the characterization of a first prototype with a $\mathrm{Pu}-\mathrm{Be}$ neutron source. In the last section, the experimental setup employed during the testing campaign in the CROCUS reactor is described. Finally, the performances of the fiber-coupled scintillator during in-core measurements are analyzed and compared with the ones of other localized neutron detection systems available at LRS, such as miniature fission chambers and an SCVD diamond detector.

\section{EXPERIMENTAL SETUP}

\section{A. Scintillator screen}

The commercially available ND2:1 scintillator screen, manufactured by Scintacor [15] and used in the present work, is 
TABLE I: Specifications of the ND2:1 scintillator screen by Scintacor [15], [16].

\begin{tabular}{|c|c|}
\hline Formula & $\mathrm{ZnS}(\mathrm{Ag}):^{6} \mathrm{LiF}$ \\
\hline Components state & Particulate blend \\
\hline Mass ratio ZnS: ${ }^{6} \mathbf{L i F}$ & $2: 1$ \\
\hline Sensitive area & $1 \mathrm{~mm}$ \\
\hline Thickness & $0.2 \mathrm{~mm}$ \\
\hline Density & $2.2 \mathrm{~g} / \mathrm{cm}^{3}$ \\
\hline${ }^{6} \mathbf{L i}$ atom density & $1.4 \times 10^{22} \mathrm{atoms}^{2} \mathrm{~cm}^{3}$ \\
\hline Peak wavelength & $450 \mathrm{~nm}$ \\
\hline Light yield & $160000 \mathrm{photons} / \mathrm{n}$ \\
\hline Decay to 10\% & $80 \mu \mathrm{s}$ \\
\hline
\end{tabular}

composed of a mixture of lithium- 6 fluoride $\left({ }^{6} \mathrm{LiF}\right)$ and zincsulphide $(\mathrm{ZnS})$ polycrystalline powders bonded with Plexiglass. The interaction between an incoming neutron and a ${ }^{6} \mathrm{Li}$ atom is described by Eq. (1). The two products of the reaction, an $\alpha$ particle and a tritium nucleus interact with the $\mathrm{ZnS}$ inorganic scintillator grains mixed into the same matrix. The scintillation light is generated as a result of the de-excitation of the luminescence centers in the $\mathrm{ZnS}$ molecules [18].

$$
{ }_{3}^{6} \mathrm{Li}+{ }_{0}^{1} \mathrm{n} \rightarrow{ }_{2}^{4} \mathrm{He}+{ }_{1}^{3} \mathrm{H} \quad \mathrm{Q}=4.78 \mathrm{MeV}
$$

An overview of the characteristics of the ND2:1 screen is reported in Tab. I. The mass ratio between the two components $\mathrm{ZnS}:{ }^{6} \mathrm{LiF}$ of $2: 1$ and the high cross section in the thermal energy region for the reaction in Eq. (1) allows reaching a good thermal neutron detection efficiency. Although ${ }^{6} \mathrm{Li}$ has a smaller thermal cross section than ${ }^{10} \mathrm{~B}$ [17], the first isotope has been preferred as a converter material for the large energy released in the reaction $(4.78 \mathrm{MeV})$ that translates into a higher light production by the $\mathrm{ZnS}$ scintillator [18]. The emitted light has a typical emission spectrum lying in the blue zone of the visible light. The scintillator sample used in the current work has a total sensitive area of $1 \mathrm{~mm}^{2}$ and a thickness of $0.2 \mathrm{~mm}$. Because of its small thickness, it shows a low intrinsic sensitivity to gamma contributions [18], which is an essential feature for its use in mixed radiations fields such as a nuclear reactor core. At the same time, the slow decay time of the emitted light by fluorescence, in the order of tens of $\mu$ s, requires signal processing techniques.

\section{B. Optical fiber}

The scintillator has been optically coupled to a polished SH8001 optical fiber, commercialized by ESKA [19], by means of the BC-630 grease of St-Gobain [20] to reduce the loss of scintillation photons. The schematic of the front end of the probe is shown in Fig. 1, where an aluminum cap protects the sensitive area of the scintillator and a black tape (visible in Fig. 2) completes the coupling with the fiber, minimizing light parasites and dispersion. In this prototype version, the optical fiber is composed of a poly(methyl methacrylate) (PMMA) core with an average diameter of $1.96 \mathrm{~mm}$ covered with a $0.04 \mathrm{~mm}$ thick fluorinated polymer cladding with a lower refractive index for light wave guiding by total internal

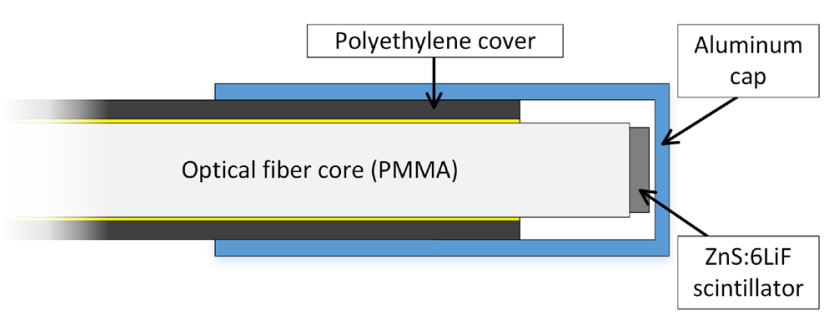

Fig. 1: Schematic of a single optical fiber front end.

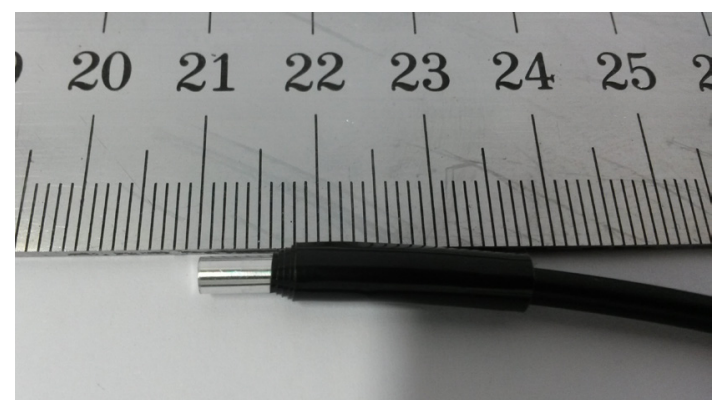

Fig. 2: Picture of a prototype of a single optical fiber coupled with $\mathrm{ZnS}:{ }^{6} \mathrm{LiF}$ scintillator, protected by an aluminum cap and wrapped with black tape.

refraction. The core refractive index is 1.49 . A $1-\mathrm{mm}$ thick jacket covers the whole length of the 10-m fiber to protect it from external damages and harsh environments.

\section{Silicon photomultiplier (SiPM)}

The rear end of the fiber was connected to a silicon photomultiplier, commonly called SiPM. A SiPM is single-photon sensitive solid-state devices made of an array of avalanches photodiodes (APDs) working in Geiger mode (called pixels and microcells) and connected in parallel on a monolithic silicon crystal [21]. In this work, the MPPC (Multi-Pixel Photon Counter) SiPM produced by Hamamatsu [21], whose specifications are described in Tab. II, was used. The SiPM was kept in the dark by being wrapped in several layers of a black cloth to avoid that external light contributions would influence the SiPM performances. A black plastic guide was designed to ensure a perfect connection between the optical fiber and the SiPM active area, minimizing parasitic light.

\section{Analog read-out electronics}

The associated analog read-out electronics is described in Fig. 3 with a detailed description of the output signals. The light photons emitted by the scintillator propagate through the optical fiber and reach the SiPM surface. The SiPM output, typically few tens of ns long, is integrated in a fast pre-amplifier designed by the LTP group at PSI. As shown in Fig. 3, the fast pre-amplifier generates a short negative output signal in the range of ns with an amplitude in the order of hundreds of $\mathrm{mV}$. A connected fast discriminator produces a square signal with a fixed amplitude when a signal corresponding to the detection of one or more photons reaches its input. This first discriminator stage is thought to cut out 


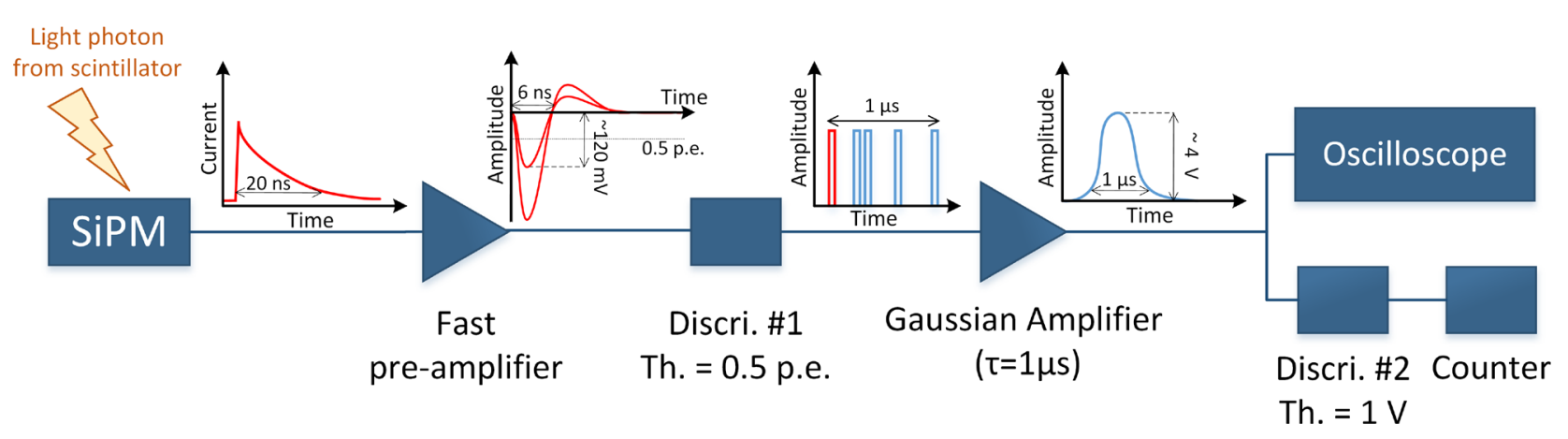

Fig. 3: Signal processing system with detailed description of the output signal after each stage.

TABLE II: Specifications of the MPPC S13360-3050PE silicon photomultiplier by Hamamatsu [21]. (*) Photon detection efficiency does not include crosstalk or after pulses.

\begin{tabular}{|c|c|}
\hline Pixel pitch & $50 \mu \mathrm{m}$ \\
\hline Effective photosensitive area & $3 \times 3 \mathrm{~mm}$ \\
\hline Number of pixels & 3,600 \\
\hline Fill factor & $74 \%$ \\
\hline Window material & Epoxy resin \\
\hline Operating temperature & -20 to $60{ }^{\circ} \mathrm{C}$ \\
\hline Spectral response range & 320 to $900 \mathrm{~nm}$ \\
\hline Peak sensitivity wavelength & $450 \mathrm{~nm}$ \\
\hline Photon detection efficiency $\left(^{*}\right)$ & $40 \%$ \\
\hline Gain & $1.7 \times 10^{6}$ \\
\hline Breakdown voltage V & $53 \pm 5$ \\
\hline Recommended operating voltage & $\mathrm{V}_{\mathrm{BR}}+3 \mathrm{~V}$ \\
\hline Crosstalk probability & $3 \%$ \\
\hline 0.5 p.e. dark count & $500 \mathrm{kcps}(\mathrm{max} .1500 \mathrm{kcps})$ \\
\hline
\end{tabular}

noise and cross-talk events in the SiPM. The result is a train of equal square pulses, each one corresponding to the detection of one or more photons at the same time. The density of pulses increases when a neutron interacts with the scintillator.

In the current work the train of pulses has been read by analog electronics. The analog system is composed by a Gaussian amplifier (CANBERRA Model 2022 [22]) generating a gaussian output signal of $1 \mu$ s width (corresponding to the amplifier shaping time), whose amplitude (in the range of few volts) depends upon the density of the train of pulses. The latter signal can be either directly read by an oscilloscope or sent to a second discriminator stage. The second discrimination, performed by a single channel analyzer (SCA) by Canberra (CANBERRA Model 2030 SCA [23]), produces fixed square signals only when the signal coming from the amplifier exceeds the defined threshold limit. The scope is to cut noise and stand-alone gamma contributions out and to count only the neutron events by means of a simple counter.

\section{PRototype CHARACTERIZATION IN CARROUSEL}

The CARROUSEL facility installed at LRS consists of a water tank containing a $\mathrm{Pu}-\mathrm{Be}$ neutron source located in its center. The aim of the test in CARROUSEL was to prove that the system composed by the miniature-size scintillator coupled

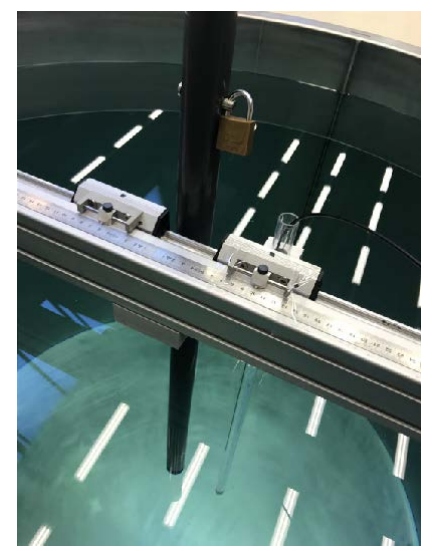

(a)

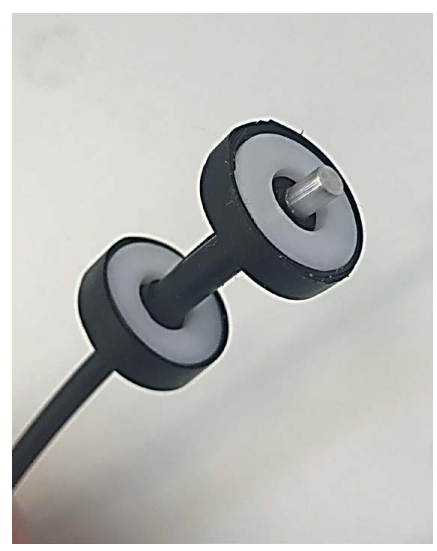

(b)
Fig. 4: (a) CARROUSEL water tank with the Pu-Be source placed at the bottom of the black tube visible in the picture. The prototype detector is placed in the transparent plexiglass tube. (b) The fiber front end with two centering tools.

to the SiPM through the optical fiber was properly designed and that the read-out mechanism was able to detect and count neutrons.

In CARROUSEL, neutrons are generated by the ${ }^{9} \mathrm{Be}(\alpha, \mathrm{n}){ }^{12} \mathrm{C}$ reaction happening in the $\mathrm{Pu}-\mathrm{Be}$ neutron source, where the $\alpha$ particles are produced by the decay of plutonium. The generated neutron rate is $8.9 \times 10^{6} \mathrm{~s}^{-1}$. Neutrons are produced with an average energy of $3.5 \mathrm{MeV}$ and a maximum energy of $10.6 \mathrm{MeV}$. In addition, the ${ }^{12} \mathrm{C}$ originating from the $(\alpha, \mathrm{n})$ reaction is generated at an excited state and thus it emits de-excitation gamma rays at $4.44 \mathrm{MeV}$.

The water tank is equipped with a rail for the placement and movement of a dedicated measurement tube in plexiglass. The fiber has been positioned in a the detector tube, as shown in Fig. 4a, and moved at different distances from the $\mathrm{Pu}-\mathrm{Be}$ source. A centering tool was used for the fiber positioning inside the tube, so that the uncertainty related to the position is $\pm 0.5 \mathrm{~mm}$. The plastic centering tools are visible in Fig. $4 \mathrm{~b}$.

The output signals from the different electronic components have been studied in detail. At first, the output signal from 


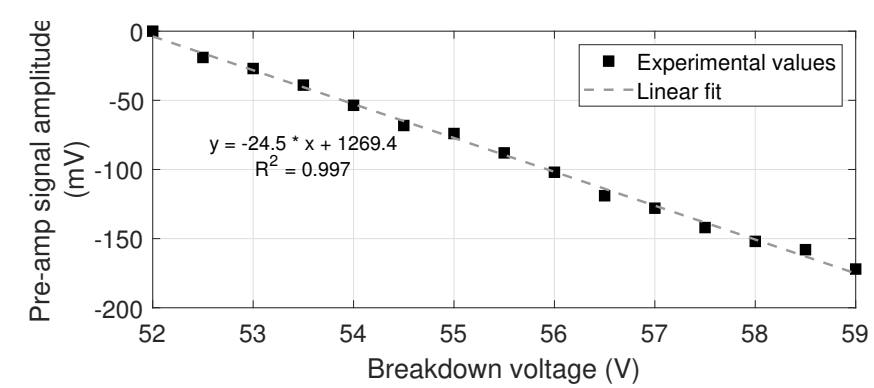

Fig. 5: Amplitude of the pre-amplifier output signal for the detection of a single photon (1 p.e.) as a function of the breakdown voltage applied to the SiPM.

the fast pre-amplifier was studied for the fiber placed at $10.00 \pm 0.05 \mathrm{~cm}$ distance from the neutron source. Secondly, the full read-out system was installed as described in the previous section and, according to the first preliminary results and to the amplifier output signal, it was properly characterized. In particular, the amplifier signal was thoroughly investigated with both the oscilloscope and the MCB multichannel analyzer produced by ORTEC [24].

\section{A. Electronic setup configuration and optimization}

The amplitude variation as a function of the breakdown voltage applied to the SiPM was recorded in order to choose the appropriate high voltage to be supplied. According to the results presented in Fig. 5, the voltage $56.50 \pm 0.05 \mathrm{~V}$ was chosen and employed in all the following measurements because it resulted in the first voltage value giving an amplitude of the output signal greater than $100 \mathrm{mV}$. The chosen value complies also with the range of recommended breakdown voltages declared by the SiPM manufacturer, as previously shown in Tab. II.

For the chosen over-voltage and the distance of $10.00 \pm 0.05 \mathrm{~cm}$, Fig. 6 shows the short $(\sim 20 \mathrm{~ns})$ negative signals exiting the fast pre-amplifier. The amplitude assumes discrete values in multiples of approximately $120 \mathrm{mV}$ per photon (p.e.). The discrete nature of the signal amplitude derives from the proportionality with the number of photodiodes triggered at the same time in the SiPM after a scintillation event, whilst each photodiode generates the same amount of charges whether being hit by single or multiple incoming photons. When a neutron interacts with the scintillator, a large quantity of light reaches the SiPM cells so that the pre-amplifier signal amplitude may reach values up to $2 \mathrm{~V}$. Figure 6 served also to determine the threshold for the first discriminator stage following the pre-amplifier. Its value was set to half the amplitude of the single photon signal $(0.5$ p.e.), corresponding approximately to $-60 \mathrm{mV}$. However, the electronic module employed to perform the first discriminator stage had a non-calibrated regulation of the threshold level. Therefore, the threshold was set testing the discriminator capabilities to digitize all the signals corresponding to one or more photon detection.

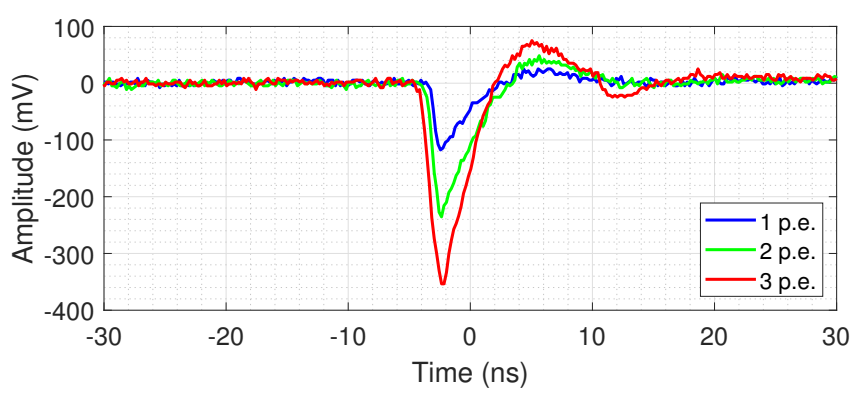

Fig. 6: Example of the output signals from the fast preamplifier recorded with the Teledyne LeCroy oscilloscope with a sampling rate of $10 \mathrm{GS} / \mathrm{s}$.

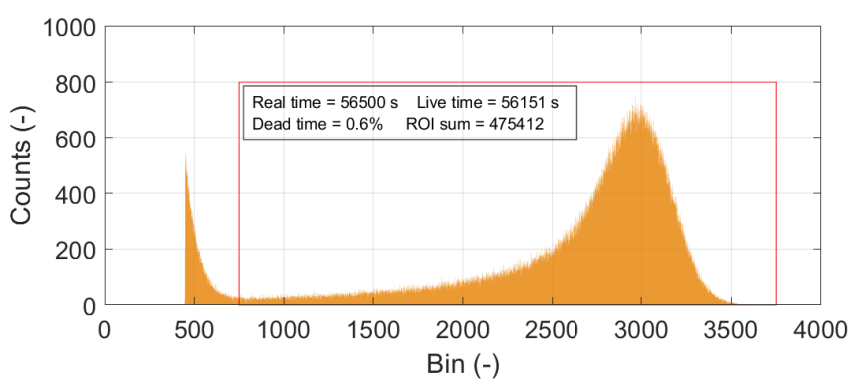

Fig. 7: Pulse height spectrum of the amplifier output signal, acquired at a distance of $10.00 \pm 0.05 \mathrm{~cm}$ distance from the $\mathrm{Pu}-\mathrm{Be}$ source. The horizontal axis is not calibrated.

Based on the knowledge acquired in the previous tests, the fast pre-amplifier output was sent to a first discriminator and an amplifier, as shown in Fig. 3. A pulse height spectrum of the amplifier output signal was recorded at the distance of $10.00 \pm 0.05 \mathrm{~cm}$ for $56500 \mathrm{~s}$ (15 hours and 41 minutes) with the MCB module connected to a computer. The results are visible in Fig. 7. Aside from the noise contribution, the spectrum shows a clear peak and a tail towards lower amplitudes given by a lower density of digital pulses, probably due to light dispersion in the fiber or in the coupling between the fiber and the SiPM. Considering a region-of-interest (ROI) starting after the noise region, the sum of the counts is $475412 \pm 689$ in a time interval of $56500 \mathrm{~s}$, the count rate being $8.414 \pm 0.012 \mathrm{cps}$. It is important to remember that the amplitude analyzed with the spectrum in Fig. 7 is proportional to the density of digital pulses generated by the first discriminator in the time interval of $1 \mu \mathrm{s}$ and thus it contributes to a small dead time. Correcting for the detector dead time, estimated to be approximately $0.6 \%$, the real count rate of events is $8.467 \pm 0.012$ cps.

The calibration of the spectrum was performed through the acquisition of 3300 signals with the Le Croy Wavesurfer 10 oscilloscope [25] and their post-processing in Matlab. With this method it is possible to reconstruct the full spectrum, as shown in Fig. 8, but the amount of data to be stored and the computational power required for the data analysis makes this approach less practical compared to the use of a MCB module. 


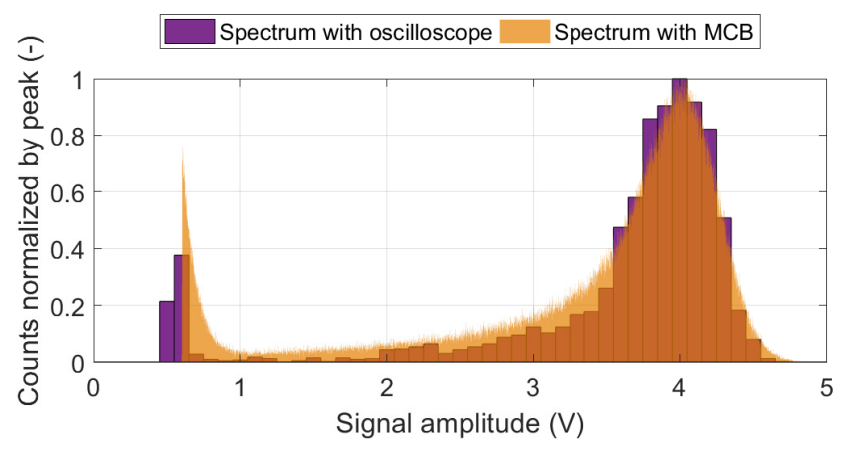

Fig. 8: Pulse height spectra obtained from oscilloscope acquisitions and from the MCB module.

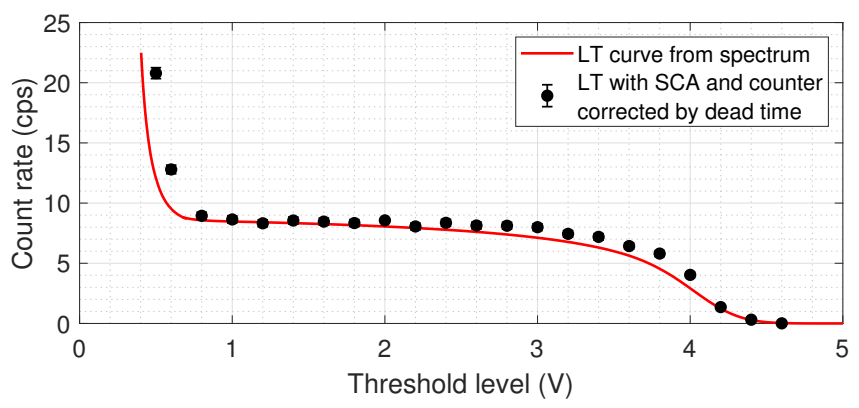

Fig. 9: Low-threshold curves reconstructed from the spectrum and acquired with a single channel analyzer (SCA) and a counter.

In addition, this method does not allow retrieving the time information and thus calculating the count rate. Nevertheless, it served for the calibration in amplitude of the spectrum that presents one peak with an average signal amplitude of $4 \mathrm{~V}$.

The lower threshold curve was reconstructed by postprocessing the spectrum acquired with the MCB and it was compared with the count rate recorded by the counter for every selected threshold of the SCA. Every signal coming from the amplifier with an amplitude above the selected threshold is transformed into a square signal that, if sent to the counter, increases the counts by one. The results, presented in Fig. 9, show a good agreement between the two sets of count rate values. Consequently, the threshold of the second discriminator stage was chosen to be $1 \mathrm{~V}$ in order to include all the events related to neutron interactions.

An example of the output signals from every component during a single neutron detection is reported in Fig. 10.

\section{EXPERIMENTAL CAMPAIGN IN THE CROCUS REACTOR}

The CROCUS reactor [14] is an experimental zero-power reactor operated at EPFL and dedicated to education and research activities. The reactor is a two-zone, uranium-fueled and light-water-moderated core. The fuel is composed of an inner uranium dioxide zone with 336 rods enriched to $1.806 \%$ and an outer uranium metal zone with 172 to 176 rods enriched to $0.947 \%$. The core height is $1 \mathrm{~m}$ and its
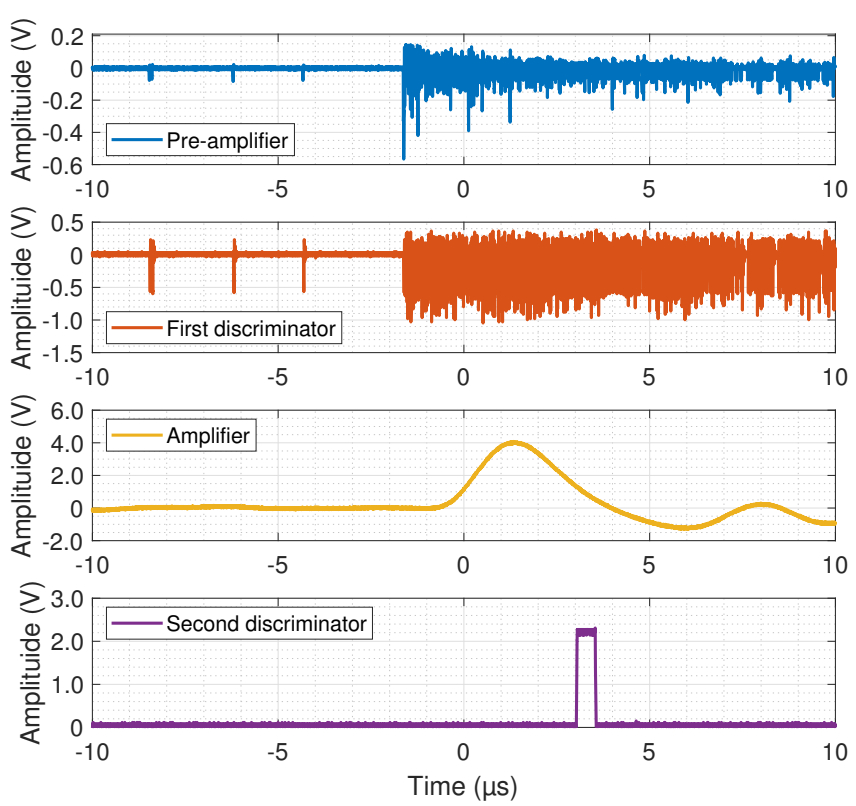

Fig. 10: Example of the output signal from each electronic component during the same neutron detection event.

diameter is around $60 \mathrm{~cm}$. The allowed fission power is limited to $100 \mathrm{~W}$, corresponding to a maximum achievable neutron flux of $2.5 \times 10^{9} \mathrm{~cm}^{-2} \cdot \mathrm{s}^{-1}$. Criticality can be achieved in two different ways in CROCUS: by adjusting the core water level and by moving the two absorbing control rods made of $\mathrm{B}_{4} \mathrm{C}$. In the following test, the first method will be used, while the control rod guide tubes will serve as experimental channels.

\section{A. Installation of the experimental setup}

The fiber front end was inserted into the guide tube of the North West control rod (see Fig. 11) of the CROCUS reactor. It was positioned so that the scintillator lies in the center of the control rod guide tube in correspondence of the active core geometrical mid-height equal to $50.0 \pm 0.5 \mathrm{~cm}$ from the lower cadmium grid (corresponding to the beginning of the fuel rods height). At this position the neutron flux is approximately at its maximum. The 10-m fiber passes through the reactor experimental channel and reaches the electronics, from the SiPM to the oscilloscope and multi-channel analyzer, placed outside the biological shield of the reactor. The setup was installed and set with the parameters previously chosen during the characterization in the CARROUSEL facility (see Section III).

The neutron count rate was acquired several times with the counter: in the shutdown configuration (i.e. without water in the reactor pool); after the insertion of the external $\mathrm{Pu}-\mathrm{Be}$ neutron source while the reactor was filled with water up to a level of $800 \pm 0.1 \mathrm{~mm}$; while the reactor was approaching its critical level by increasing the water level. Once the reactor reached its critical configuration, the neutron count rate was estimated at different power levels to test the linearity of the response of the detector with an increasing reactor power. 


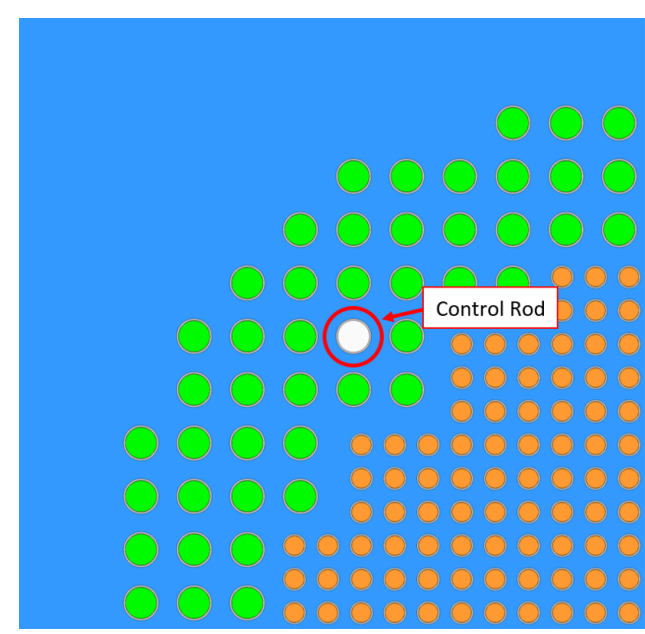

Fig. 11: Horizontal cut of the CROCUS reactor schematic (taken from the model in Serpent 2) with indication of the position for the fiber placement.

TABLE III: Neutron count rate in CROCUS after the neutron source insertion, while the water was filled up to $800 \pm 0.1 \mathrm{~mm}$.

\begin{tabular}{|c|c|}
\hline $\begin{array}{c}\text { Time after source } \\
\text { insertion (min) }\end{array}$ & Count rate (cps) \\
\hline 0 & $0.38 \pm 0.06$ \\
\hline 2 & $3.00 \pm 0.17$ \\
\hline 5 & $5.5 \pm 0.2$ \\
\hline 7 & $6.0 \pm 0.2$ \\
\hline 9 & $6.0 \pm 0.2$ \\
\hline
\end{tabular}

\section{B. Results and discussion}

Before conducting the measurements in the reactor at power, a background acquisition with the reactor in shutdown state has been performed. It has been measured that the background count rate in the CROCUS cavity (in the control rod position) is equal to $1.7 \times 10^{-4} \mathrm{cps}$ with a $20 \%$ statistical error. This incore blank measurement lasted for 38.5 hours and it suggested that the detection system is not counting events related to background radiation or fuel activation.

Once the external neutron source was inserted, the neutron count rate (measured with the counter) started to increase due to the water filling-up procedure, until a stable configuration was reached at the water level of $800 \pm 0.1 \mathrm{~mm}$ (see Tab. III). The water level was further increased in steps to approach and determine the reactors critical level. The measured count rate is reported in Tab. IV. The final critical water level was set by the operator at $957.8 \pm 0.1 \mathrm{~mm}$ for a power of $40 \mathrm{~mW}$. At this power, the count rate acquired with the counter is $168.4 \pm 1.0 \mathrm{cps}(0.6 \%$ error $)$.

The linearity of the detector response has been tested increasing the reactor power from $40 \mathrm{~mW}$ up to $10.8 \mathrm{~W}$, corresponding approximately to the range from $3.8 \times 10^{5}$ to $1.0 \times 10^{8} \mathrm{~cm}^{-2} \cdot \mathrm{s}^{-1}$ in the detector position. The count rate acquired with the counter is reported in Tab. V and
TABLE IV: Neutron count rate in CROCUS during the approach to critical. The water level is increased in steps until the critical level was found.

\begin{tabular}{|c|c|}
\hline Water level $(\mathbf{m m})$ & Count rate (cps) \\
\hline $910.0 \pm 0.1$ & $22.2 \pm 0.3$ \\
\hline $920.0 \pm 0.1$ & $28.3 \pm 0.2$ \\
\hline $933.0 \pm 0.1$ & $43.1 \pm 0.4$ \\
\hline $941.0 \pm 0.1$ & $63.5 \pm 0.6$ \\
\hline Critical $40 \mathrm{~mW}$ & $168.4 \pm 1.0$ \\
\hline
\end{tabular}

TABLE V: Neutron count rate acquired at different power levels of the CROCUS reactor.

\begin{tabular}{|c|c|c|}
\hline Power (W) & Count rate (kcps) & Error \\
\hline 0.125 & $0.441 \pm 0.002$ & $0.5 \%$ \\
\hline 0.2 & $0.677 \pm 0.003$ & $0.5 \%$ \\
\hline 0.5 & $1.717 \pm 0.007$ & $0.4 \%$ \\
\hline 0.8 & $2.778 \pm 0.010$ & $0.4 \%$ \\
\hline 1 & $3.368 \pm 0.018$ & $0.5 \%$ \\
\hline 1.25 & $4.37 \pm 0.02$ & $0.5 \%$ \\
\hline 1.57 & $5.42 \pm 0.02$ & $0.4 \%$ \\
\hline 2.035 & $6.99 \pm 0.03$ & $0.4 \%$ \\
\hline 3 & $10.33 \pm 0.03$ & $0.3 \%$ \\
\hline 4.9 & $16.57 \pm 0.04$ & $0.2 \%$ \\
\hline 6.5 & $21.35 \pm 0.05$ & $0.2 \%$ \\
\hline 8.85 & $25.48 \pm 0.05$ & $0.2 \%$ \\
\hline 10.8 & $26.70 \pm 0.05$ & $0.2 \%$ \\
\hline
\end{tabular}

plotted in Fig. 12 as a function of the reactor power. The reactor power is obtained from the out-core monitor fission chamber of CROCUS, which are calibrated using the gold foil activation technique [26]. The evolution of the count rate shows a good capability of the system to linearly respond to the increase of the reactor power up to $6.5 \mathrm{~W}$, corresponding to approximately $6.2 \times 10^{7} \mathrm{~cm}^{-2} \cdot \mathrm{s}^{-1}$ in the North West control rod guide tube at core mid-height. The subsequent loss of linearity is caused by common detection dead time, given by the fact that analog signal processing is employed by the readout electronics. Indeed, the Gaussian amplifier processes the pulses reaching its input in the time interval of $1 \mu$ s and thus trains of pulses deriving from different neutron interactions might be read together contributing to a single amplifier pulse. This effect becomes relevant at higher reactor power, where the neutron count rate increases significantly. This limitation might be partially overcome by reducing the shaping time of the gaussian amplifier to have a better discrimination between different neutron events at higher neutron flux rates.

\section{Comparison with different in-core instrumentation}

Other measurements in the same position and irradiation conditions, i.e. criticality and same neutron spectrum, have been performed in the past in CROCUS with different types of neutron detectors. All the neutron detectors have been placed in the guide tube of the North West control rod of the CROCUS reactor, at approximately $50 \mathrm{~cm}$ from the bottom of the core active zone. The neutron count rate shown by a $\mathrm{BF}_{3}$ detector (type Transcommerce $\mathrm{MN}-1$ ) in the aforementioned position is about $180000 \mathrm{cps} / \mathrm{W}$ [27], whereas 


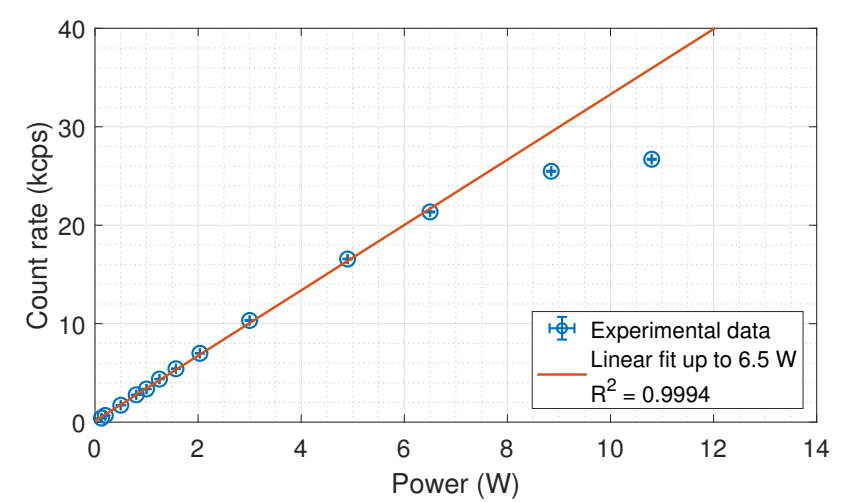

Fig. 12: Neutron count rate in CROCUS as a function of the reactor power. Data are linearly fitted up to $6.5 \mathrm{~W}$.

TABLE VI: Neutron count rate per unit power in the North West control rod guide tube of CROCUS for different neutron detection technologies [27], [28].

\begin{tabular}{|c|c|}
\hline Detector type & $\begin{array}{c}\text { Neutron count rate per } \\
\text { unit power }(\mathbf{k c p s} / \mathbf{W})\end{array}$ \\
\hline Cividec sCVD & 0.15 \\
\hline Photonis MFC & 0.56 \\
\hline Scintillator + fiber & 3.37 \\
\hline Transcommerce $\mathrm{BF}_{3}$ & 180 \\
\hline
\end{tabular}

the value registered by the Cividec SCVD diamond detector is $150 \mathrm{cps} / \mathrm{W}$ [28] and a miniature fission chamber (MFC, Photonis CFUR44/EP-M3 type with $130 \mu \mathrm{g}$ of ${ }^{235} \mathrm{U}$ ) displays $560 \mathrm{cps} / \mathrm{W}$. In the same position, the detection setup composed by the scintillator screen coupled with the optical fiber and the described read-out electronics gives a neutron count rate equal to $3368 \mathrm{cps} / \mathrm{W}$. The results are summarized in Tab. VI.

Despite the limitations due to the read-out electronics (as described before), the neutron count rate obtained with the system designed in the current work is very high for such a small scintillator volume ( $1 \mathrm{~mm}^{2}$ area and $0.2 \mathrm{~mm}$ thickness), which makes this technology of interest for the investigation of localized and three-dimensional phenomena in research reactors. The detector shape and arrangement might be also designed in order to increase the detection efficiency and to optimize the light collection. In addition, the use of the advanced digital electronics (currently under testing) will allow parallelizing the designed detector and to perform multichannel online measurements.

\section{CONCLUSIONS}

The current test proved the feasibility of neutron detection with a $\mathrm{ZnS}:^{6} \mathrm{LiF}$ screens coupled to silicon photomultiplier (SiPMs) via optical fibers in mixed neutron and gamma fields. The miniature-size detector and its read-out electronics have been thoroughly characterized in the CARROUSEL facility. The detection system, tested in the CROCUS zero-power research reactor, showed a linear behaviour up to the power of $6.5 \mathrm{~W}$ (in order of $\sim 10^{8} \mathrm{~cm}^{-2} \cdot \mathrm{s}^{-1}$ total neutron flux). It also shows an excellent neutron sensitivity with respect to other instruments of reduced size such as miniature fission chambers and SCVD diamond detectors, both available at LRS. The lack of detected events in the reactor shutdown configuration suggests a good gamma-blindness of the system that will be further investigated. The first testing campaign proved that the fiber-coupled scintillator system developed and tested in this work is an efficient and practical solution for the investigation of complex in-core phenomena. The excellent performances combined with its simplicity will allow us to use it for multiple simultaneous localized measurements in the CROCUS reactor. Additional developments and tests are foreseen to be performed in the future in order to fullycharacterize the miniature detector, improve its performances and broaden its applications in the CROCUS reactor.

\section{REFERENCES}

[1] C. Pralong Fauchre, O. Joneja, R. Chawla, P. Grimm, F. Jatuff and M. Murphy, "Reactor analysis methods 5 Within-Pin Reaction Rate Distributions in a SVEA-96+ Fuel Assembly," Transactions of the American Nuclear Society, 211-212, 2001.

[2] C. Pralong Fauchre, M. Murphy, F. Jatuff and R. Chawla, "Within-pin Reaction Rate Distributions: CASMO-4 and HELIOS Compared Against Tomographic Measurements at the PROTEUS Reactor," Nuclear Science and Engineering, 150:1, 27-36, 2005.

[3] K. Macku, F. Jatuff, M. Murphy, M. Plaschy, P. Grimm, O. P. Joneja and R. Chawla, "Radial and Azimuthal ${ }^{235} \mathrm{U}$ Fission and ${ }^{238} \mathrm{U}$ Capture Distributions in BWR UO 2 Pins: CASMO-4 and MCNP4C versus Activation Foil Measurements," Nuclear Science and Engineering, 155:1, 96-101, 2007.

[4] M. F. Murphy, M. Plaschy, F. Jatuff, U. Bergmann and R. Chawla, "Fission and capture rate measurements in a SVEA-96 OPTIMA2 BWR assembly compared with MCNPX predictions," United States: American Nuclear Society - ANS, 2006.

[5] V. Lamirand, M. Hursin, P. Frajtag, G. Perret, O. Pakari and A. Pautz, "Future experimental programmes in the CROCUS reactor," European Research Reactor Conference (RRFM) 2016: Conference Proceedings, (p. 1154). Belgium: European Nuclear Society, 2016.

[6] F. Vitullo, V. Lamirand and A. Pautz, "A novel neutron detector for localized in-core measurements in the CROCUS reactor for high-fidelity code validation purposes." The poster will be presented at the Poster session at the FISA2019 conference, 4-7 June 2019, Pitesti, Romania.

[7] R. T. Kouzes, A. T. Lintereur, and E. R. Siciliano, "Progress in alternative neutron detection to address the helium-3 shortage," Nucl. Instruments Methods Phys. Res. Sect. A Accel. Spectrometers, Detect. Assoc. Equip., vol. 784, pp. 172-175, Jun. 2015.

[8] C. Mori, T. Osada, K. Yanagida, T. Aoyama, A. Uritani, H. Miyahara, Y. Yamane, K. Kobayashi, C. Ichihara and S. Shiroya, "Simple and Quick Measurement of Neutron Flux Distribution by Using an Optical Fiber with Scintillator," Journal of Nuclear Science and Technology, 31:3, 248-249, 1994.

[9] Y. Yamane, A. Uritani, and T. Misawa, "Measurement of the thermal and fast neutron flux in a research reactor with a $\mathrm{Li}$ and $\mathrm{Th}$ loaded optical fibre detector," Nucl. Instruments Methods Phys. Res. Sect. A Accel. Spectrometers, Detect. Assoc. Equip., vol. 432, pp. 403-409, 1999.

[10] M. Stagliano, A. Chierici, L. Abego, and F. dErrico, "Silicon photomultiplier current and prospective applications in biological and radiological photonics," se, vol. 4, no. 10, pp. 10-29, Oct. 2018.

[11] J.-B. Mosset, A. Stoykov, U. Greuter, A. Gromov, M. Hildebrandt, T. Panzner and N. Schlump, "A 16-ch module for thermal neutron detection using ZnS:6LiF scintillator with embedded WLS fibers coupled to SiPMs and its dedicated readout electronics," Nucl. Instruments Methods Phys. Res. Sect. A Accel. Spectrometers, Detect. Assoc. Equip., vol. 845, pp. 494-498, 2016.

[12] A. Stoykov, J. -B. Mosset, and M. Hildebrandt, "Trigger Efficiency of a ZnS:6LiF Scintillation Neutron Detector Readout With a SiPM," IEEE Trans. Nucl. Sci., vol. 63, no. 4, pp. 2271-2277, 2016. 
[13] International Atomic Energy Agency, "Advanced Surveillance, Diagnostic and Prognostic Techniques in Monitoring Structures, Systems and Components in Nuclear Power Plants," IAEA Nuclear Energy Series No. NP-T-3.14, Vienna, 2013.

[14] V. Lamirand, "Installation nucléaire CROCUS - Rapport de sécurité version 1.2," 2017.

[15] Scintacor, "Neutron Screens,". [Online]. Available: https://scintacor.com/wp-content/uploads/2015/09/Datasheet-NeutronScreens-High-Res.pdf

[16] C. W.E van Eijk, "Neutron PSDs for the next generation of spallation neutron sources," Nuclear Instruments and Methods in Physics Research Section A: Accelerators, Spectrometers, Detectors and Associated Equipment, Volume 477, Issues 13, 2002, Pages 383-390.

[17] N. Soppera, M. Bossant and E. Dupont, "JANIS 4: An Improved Version of the NEA Java-based Nuclear Data Information System," Nuclear Data Sheets, Volume 120, June 2014, Pages 294-296.

[18] G.F. Knoll, "Radiation Detection and Measurement," 4th edition, 2010.

[19] ESKA, "Specification sheet SH-8001," 2001. Available: http://www.ifiberoptics.com/pdf/sh8001.pdf.

[20] Saint-gobain, "Safety data sheet BC-630 Silicone Grease," 2017. Available: https://www.crystals.saintgobain.com/sites/imdf.crystals.com/files/documents/bc-630.pdf

[21] A. Ghassemi, K. Sato, and K. Kobayashi, "MPPC - Technical note," Hamamatsu Photonics K.K., Solid State Division, 2017. Available: https://www.hamamatsu.com/resources/pdf/ssd/mppc_kapd9005e.pdf

[22] CANBERRA, "Model 2022 Spectroscopy Amplifier," 2010. Available: https://www3.nd.edu/ wzech/Model-2022-SS-M3833.pdf

[23] CANBERRA, "Model 2030 SCA." Available: http://web.mit.edu/8.13/8.13d/manuals/canberra/canberra-2030-sca.pdf

[24] ORTEC, "926-M32-USB Multichannel Analyzer," 2015. Available: http://www-old.pd.infn.it/elettronica/Strumenti/926-m32-usb.pdf

[25] TELEDYNE LECROY, "WaveSurfer 10 oscilloscope," 2015. Available: http://cdn.teledynelecroy.com/files/pdf/wavesurfer10-datasheet.pdf

[26] V. Lamirand, O. Pakari and A. Pautz, "Power calibration methodology at the CROCUS reactor, " presented at ANIMMA2019 conference, 17-21 June 2019, Portoroz, Slovenia.

[27] G. Perret, G. Girardin, P. Frajtag, and M. Hursin, "Decay constant and delayed neutron fraction measurements in CROCUS," Paul Scherrer Institut, TM-41-14-02 Rev. 1, 2014.

[28] M. Hursin, C. Weiss, P. Frajtag, V. Lamirand, G. Perret, P. Kavrigin, A. Pautz, and E. Griesmayer, "Testing of a sCVD diamond detection system in the CROCUS reactor," Eur. Phys. J. A, 54: 82, 2018. 